\title{
Change in sagittal profile after implantation of anchored interbody cage in the surgical procedure for degenerative cervical spine disease
}

\author{
Rene Opsenak ${ }^{\mathrm{a}}$, Martin Hanko ${ }^{\mathrm{a}}$, Pavol Snopko ${ }^{\mathrm{a}}$, Martin Benco ${ }^{\mathrm{a}}$, Radoslav Hanzel ${ }^{\mathrm{a}}$, Branislav Kolarovszkia
}

Background. The aim of this study was to verify the relationship between changes in the segmental sagittal profile (SSP) and changes in the global sagittal profile (GSP) after anterior cervical discectomy with anchored cage implantation (ACDF).

Study design. Prospective study with 2-year follow-up.

Methods. This study includes 104 patients after 1-level or 2-level ACDF operated between the May 2013 and March 2016. SSP was evaluated by Cobb angle measurement of operated motion segment (CobbS) and GSP was evaluated by Cobb angle measurement in C2-C7 segments (CobbG). Both SSP and GSP were measured pre- and postoperatively within a 24 months follow-up period. The influence of factors such as age, gender, number of treated segments and osteoporosis was evaluated using t-tests. The correlation between SSP and GSP changes was assessed by Pearson's correlation coefficient.

Results. In the early postoperative period after 1-level ACDF, there was a significantly greater increase in CobbS compared to that of the 2-level ACDF $(P=0.0149)$. Male patients experienced a significant decrease of CobbG during the first 6 months after surgery as well as patients with osteoporosis within 12 months after ACDF. After ACDF the SSP change weakly correlated with the GSP change.

Conclusion. SSP change after 1- or 2-level ACDF correlates mostly weakly with GSP change. Male gender and osteoporosis were identified as risk factors for global lordotisation following ACDF.

Key words: anterior cervical discectomy, sagittal profile, risk factors, anchored cage

Received: November 6, 2019; Revised: February 19, 2020; Accepted: February 26, 2020; Available online: March 9, 2020

https://doi.org/10.5507/bp.2020.007

(c) 2021 The Authors; https://creativecommons.org/licenses/by/4.0/

${ }^{a}$ Clinic of Neurosurgery, Jessenius Faculty of Medicine in Martin, Comenius University in Bratislava, Slovak Republic Corresponding author: Branislav Kolarovszki, e-mail:branislav.kolarovszki@uniba.sk

\section{INTRODUCTION}

Anterior cervical discectomy and fusion (ACDF) is one of the most common surgical procedures in the treatment of the degenerative cervical spine disease. The aim is to decompress the spinal canal and spinal nerves located in the degenerated motion segment. Implantation of a cervical interbody cage allows for restoration of the intervertebral space height, induction of interbody fusion and lordotisation of the operated motion segment. However, the intraoperative lordotisation of the motion segment may subsequently regress. The effect of the segmental sagittal profile correction (SSP) on the development of the global sagittal profile (GSP) of the cervical spine is variable and influenced by various factors.

\section{MATERIALS AND METHODS}

Our prospective study includes 104 patients with degenerative cervical spine disease. All of these patients underwent a 1- or 2-level ACDF with an implantation of Zero Profile Variable Angle ${ }^{\circledR}$ cage (Zero-P VA ${ }^{\circledR}$, DePuy Synthes, Switzerland, Fig. 1.). ACDF was indicated based on clinical and graphical findings after 6 weeks of un- satisfactory conservative treatment. In all cases, motion segments with graphical signs of osteochondrosis were indicated for treatment. The exclusion criteria for this study were defined as: presence of myelopathy, pregnancy, inflammatory or malignant disease of the cervical spine, injuries of the cervical spine and general contraindications of elective surgery. According to age they were divided into two groups - patients younger than 55 years of age and patients aged 55 years and more. Similarly, our cohort was divided according to sex, number of operated cervical motion segments and osteoporosis (which was confirmed by means of bone densitometry). All patients underwent a cervical spine X-ray examination in anterior and lateral projections before surgery, in early postoperative period (up to $48 \mathrm{~h}$ after ACDF), at 6 weeks, 3 months, 6 months, 12 months and 24 months after ACDF. The SSP was evaluated by means of measurement of the Cobb angle between the upper body surface of proximal and the lower body surface of the distal vertebra of the motion segment (CobbS). The GSP was similarly evaluated using the measurement of the Cobb angle between the lower vertebral body of $\mathrm{C} 2$ and the lower vertebral body of $\mathrm{C} 7$ (CobbG) or the most distally visualized vertebra above the $\mathrm{C} 7$, but always in the same manner for a particular patient (Fig. 2.). Early postoperative changes in sagittal profile 


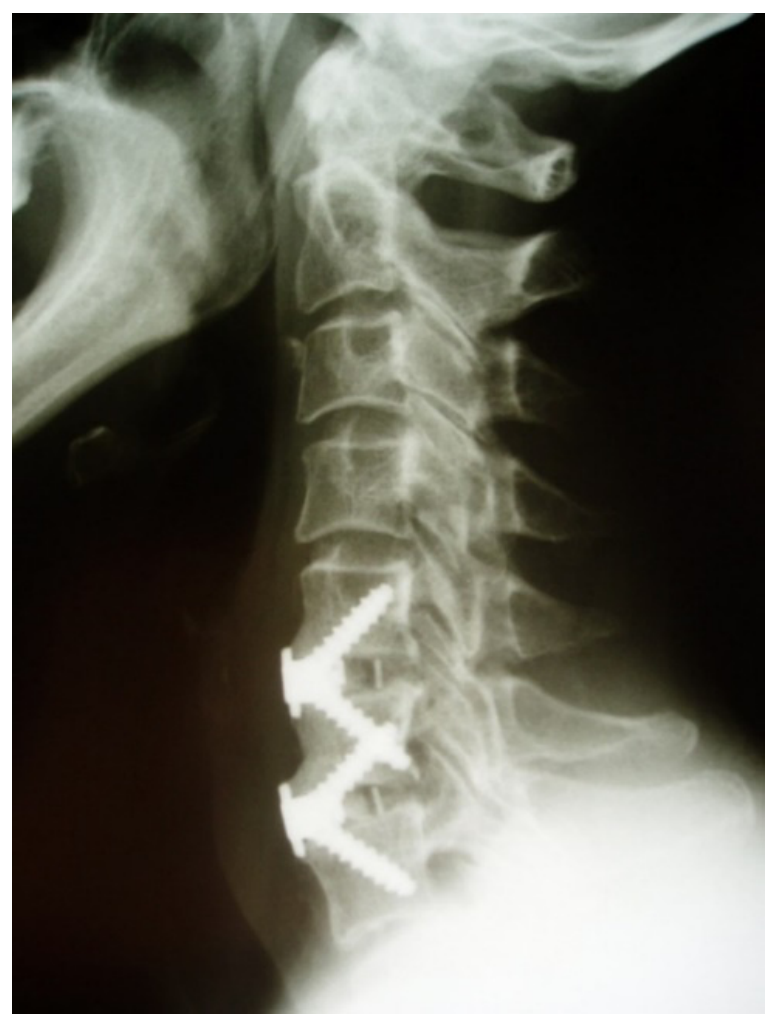

Fig. 1. Lateral X-ray after ACDF with Zero-P VA ${ }^{\circledR}$ cage in levels C5-6, C6-7 (authors' archive).

were then evaluated as the difference between CobbS (CobbG) values in the preoperative and early postoperative period (up to $48 \mathrm{~h}$ ). Differences in SSP and GSP during the further postoperative period were evaluated as the difference between CobbS (CobbG) values in the early postoperative period and CobbS (CobbG) values over 6 weeks, 3 months, 6 months, 12 months and 24 months after ACDF. Statistical significance of factors such as age, gender, number of operated motion segments and osteoporosis was evaluated using Student's unpaired t-test. The impact of the cage subsidence (CS) on CobbS value in term of 24 months after ACDF was evaluated using the same test. Correlation between SPP and GSP changes was assessed by means of the Pearson's correlation coefficient (r). The Cohen scheme was used to interpret Pearson correlation coefficient - absolute values up to 0.1 represented a trivial correlation, values between $0.1-0.3$ represented a weak correlation, values $0.3-0.5$ represented a moderate correlation and values above 0.5 represented a strong correlation ${ }^{1}$.

\section{RESULTS}

We performed ACDF with the implantation of the anchored Zero-P VA ${ }^{\circledR}$ cage in an overall of 159 motion segments. In 49 patients it was a 1-level ACDF and in 55 it was a 2-level ACDF. The level C5/6 was treated most frequently (Table 1). Table 2 depicts the division of the cohort according to age, gender, number of treated mo-

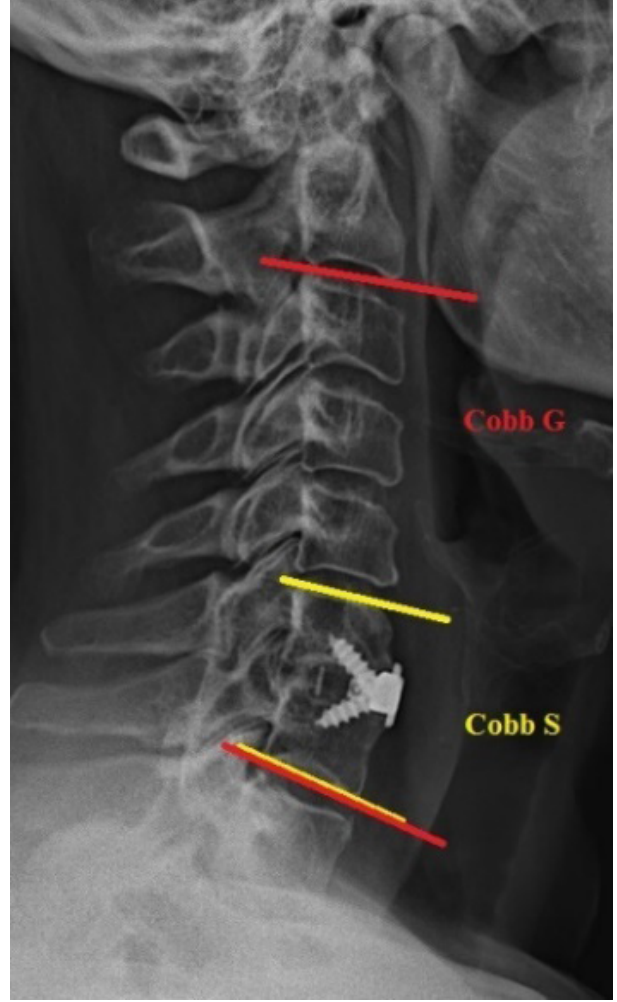

Fig. 2. The method of evaluation of CobbS and CobbG (authors' archive).

tion segments and presence of densitometrically verified osteoporosis.

The an average preoperative value of CobbS was $1.45^{\circ}$, representing a lordotic SSP. Age, gender and osteoporosis had no significant effect on the SSP according to preoperative findings. In the early postoperative period, the average CobbS was increased by $319.3 \%\left(4.63^{\circ}\right)$. In females, patients younger than 55 years and non-osteoporotic patients the acquired segmental lordotisation was bigger, however the differences were not statistically significant $(P=0.05)$. Patients after 1-level ACDF presented with a significantly higher increase of the CobbS value than the CobbS increase in patients after the 2-level ACDF $(P=0.0149) .6$ weeks after the ACDF an average decrease of CobbS by $16.6 \%$ was observed, 3 months postoperatively the decrease reached $27.6 \%$ with a further gentle correction - a total decrease of $27.3 \%$ in 6 months postoperatively. The average value of CobbS decreased by

Table 1. Representation of treated motion segments in our patients' cohort.

\begin{tabular}{lcc}
\hline Segment & $\begin{array}{c}\text { Absolute } \\
\text { number }\end{array}$ & $\begin{array}{c}\text { Percentual } \\
\text { representation (\%) }\end{array}$ \\
\hline C2/3 & 0 & 0 \\
C3/4 & 10 & 6.3 \\
C4/5 & 35 & 22.0 \\
C5/6 & 65 & 40.9 \\
C6/7 & 42 & 26.4 \\
C7/T1 & 7 & 4.4 \\
\hline
\end{tabular}


Table 2. Division of the patients' cohort according to analysed risk factors.

\begin{tabular}{llll}
\hline Age & Gender & Number of treated segments & Osteoporosis \\
\hline Up to 55 years: 65 & Men: 48 & 1 segment: 49 & Confirmed: 41 \\
55 years and older: 39 & Women: 56 & 2 segments: 55 & Unconfirmed: 63 \\
\hline
\end{tabular}

Table. 3. Correlation between SSP and GSP development after ACDF according to Pearson's coefficient.

\begin{tabular}{lc}
\hline $\begin{array}{l}\text { Time period } \\
\text { after ACDF }\end{array}$ & $\begin{array}{c}\text { Correlation between } \\
\text { CobbS a CobbG (r) }\end{array}$ \\
\hline first $48 \mathrm{~h}$ & 0.281 \\
6 weeks & 0.238 \\
3 months & 0.408 \\
6 months & 0.322 \\
12 months & 0.271 \\
24 months & 0.278 \\
\hline
\end{tabular}

$30 \%$ after 12 postoperative months and by $40.9 \%$ after 24 postoperative months. We were unable to confirm any effect of gender, age, osteoporosis or number of treated segments on the decrease in lordotisation in the period of 6 weeks to 24 months post $\operatorname{ACDF}(P=0.05) .24$ months after the ACDF the average value of the CobbS was increased by $2.1^{\circ}(144.8 \%)$. Subsidence of the anchored cage more than $3 \mathrm{~mm}$ into adjacent vertebral bodies in term of 24 months after ACDF occurred in 13 motion segments (8.2\%). CS had no significant impact on CobbS value in that period $(P=0.2005)$. In all cases, $C S$ was found in the ventral portion of the motion segment. Number of treated motion segments in the patient had no significant impact on the incidence of $\operatorname{CS}(P=0.7692)$.

The average preoperative value of CobbG was $7.5^{\circ}$ with more lordotic values being observed in male patients, however, this difference was not statistically significant. Patients aged 55 years and more presented with a significantly higher average value of the CobbG than younger patients $(P=0.0178)$. Osteoporotic patients also presented with a significantly higher preoperative CobbG than patients without this particular comorbidity $(P=0.0011)$. In the early postoperative period, the average value of CobbG was increased by $34.8 \%$. In contrast to female patients, in the male group we observed a significant early decrease of the CobbG $(P=0.0014)$. Age, osteoporosis and number of operated segments did not affect the early postoperative GSP $(P=0.05)$. Six weeks after the ACDF, the average CobbG was increased by $8.8 \%, 3$ months postoperatively the early obtained average value of CobbG decreased by $2.8 \%$. The average CobbG was later increased - by $21.6 \%$ after 6 postoperative months and by $8.6 \%$ after 12 postoperative months. 24 months after the ACDF the average CobbG was larger by $1.8 \%$ only compared to early postoperative findings, but increased by $25.7 \%$ compared to preoperative findings. After a 1-level ACDF the global lordotisation was subsequently lost. In contrast, the global lordotisation increased in the further postoperative period after the 2-level ACDF compared with the early postoperative values, however these differences were not significant $(P=0.05)$. The female patients present with an increase in average postoperative $\mathrm{CobbG}$, while an opposite trend was recorded in male patients. These differences were significant at 6 weeks, 3 months and 6 months after the ACDF $(P<0.0298)$, but not significant in the following period $(P=0.05)$. In osteoporotic patients, the early rec-

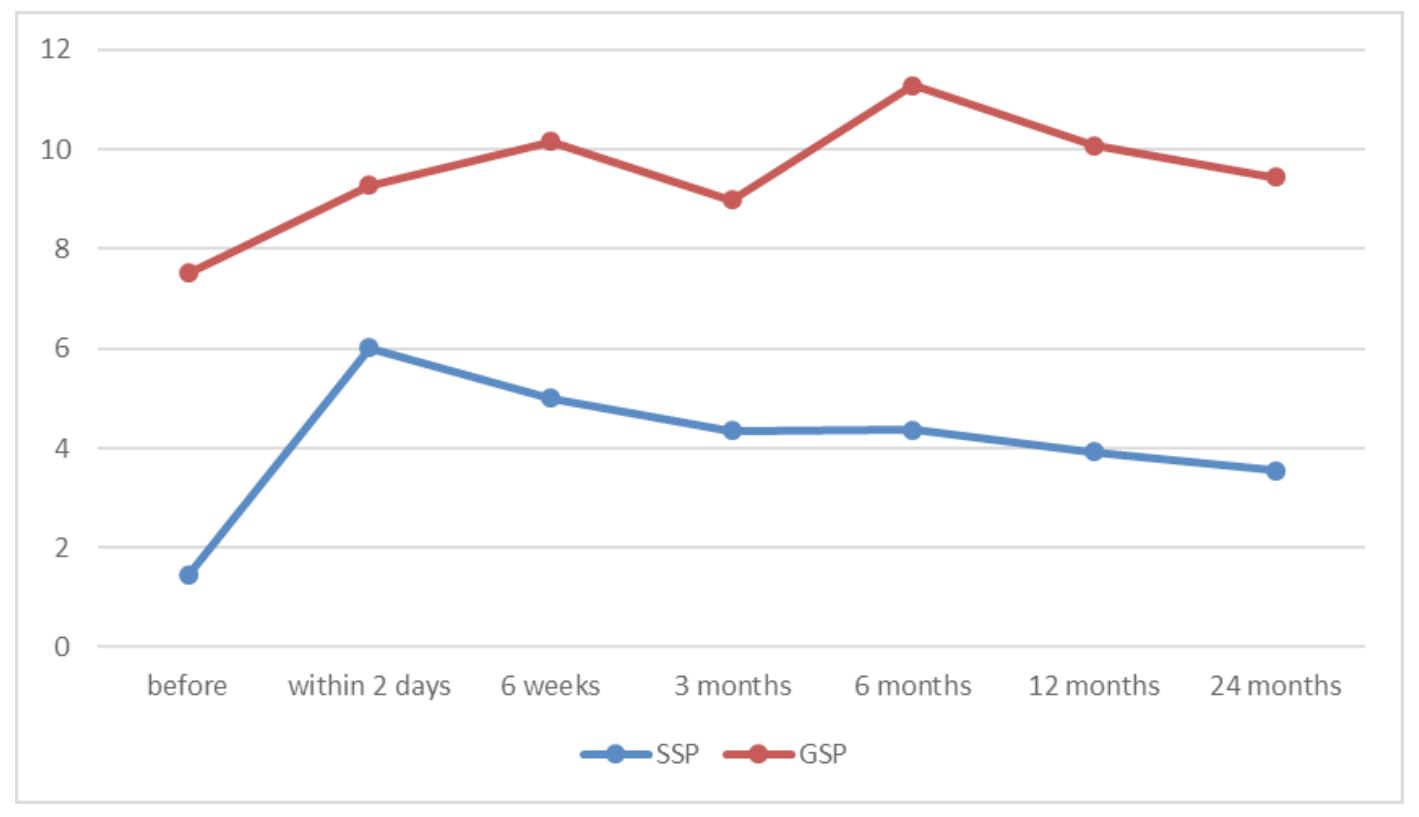

Fig. 3. The trend graph of SSP and GSP development after the ACDF (represented in degrees). 
ognised global lordotisation was significantly decreased at 12 months after the $\operatorname{ACDF}(P=0.0354)$, the differences were, however, not significant during the other imaging controls $(P=0.05)$. Patient age did not significantly affect the dynamics of the average postoperative CobbG in 6 weeks to 24 months after the $\operatorname{ACDF}(P=0.05)$.

In the early postoperative period both global and segmental lordotisation was increased. However, in the further postoperative X-ray controls the segmental lordotisation was observed to be decreasing while the GSP did not follow this trend (Fig. 3.). 24 months after the ACDF the average value of the CobbS was lower than the preoperative value. In contrast the average CobbG was still increased. The correlation between the values of the CobbS and CobbG was moderate at 3 and 6 months postoperatively and weak in 6 weeks, 12 and 24 months after the ACDF (Table 3).

\section{DISCUSSION}

In our study, a single type of an anchored zero profile interbody cage was used, specifically Zero-P VA ${ }^{\circledR}$. This implant is fixed by inserting two divergently directed screws introduced into the adjacent vertebral bodies. The cage design assumes an elimination of disadvantages of the conventional cervical plates and maintenance of their benefits $^{2,3}$. Stated advantages of anchored interbody cages include a reduction of postoperative adhesions, reduction of postoperative dysphagia, smaller surgical approach, shorter duration of surgery and a prevention of adjacent segment ossification when compared to those after the use of cages fixed by the conventional plate $\mathrm{p}^{3-10}$.

We observed an early lordotisation of the treated motion segment, which was more significant after 1-level ACDF. During the further postoperative period, a reduction of the surgically-acquired segmental lordotisation occurred. The change was however not affected by factors such as gender, age, osteoporosis or number of treated segments. The design of the interbody cage used in our study (Zero-P VA ${ }^{\circledR}$ ) is similar to that of a Zero-P ${ }^{\circledR}$ implant. A number of studies describe a loss of surgicallyacquired segmental lordotisation after the implantation of Zero- $\mathrm{P}^{\circledR}$ cage $^{3,9-14}$. Fixation of Zero-P VA ${ }^{\circledR}$ cage ( similar to the Zero- ${ }^{\circledR}$ cage) is provided by using divergent screws and causes a certain degree of segmental distraction in the ventral portion of the treated motion segment, especially during the early postoperative period after ACDF. A greater lordotising effect therefore occurs compared to the one obtained by a cage fixation with the conventional plate. Also, when the conventional plate is used, a compression of the ventral portion of intervertebral space occurs, causing a reduction of the segmental lordotic profile $\mathrm{e}^{3,13,14}$. During the further postoperative period the surgically-acquired segmental lordotisation tends to reduce due to the effect of axial loading and also due to a lower biomechanical stability of anchored cages, especially in anteflexion and retroflexion ${ }^{2}$. When a conventional cervical plate is used for stabilisation, the physiological mechanical load- ing acts against the plate's tension ${ }^{3}$. Several studies have reported a significantly lower segmental lordotisation after Zero- $\mathrm{P}^{\circledR}$ cage implantation compared to that obtained by using a cage fixed by a conventional plate ${ }^{9,10}$. Likewise Zhang et al., we have not confirmed any effect of osteoporosis on the SSP development after the implantation of the anchored cage ${ }^{15}$. Anchored interbody cages fixed with divergently inserted screws are therefore safe to use even in the osteoporotic regions. A more significant segmental lordotisation obtained after 1-level ACDF was characteristic for the whole cohort of our patients. We believe that this is a result of a tendency of the operating surgeon to obtain a greater lordotisating effect when operating in a single motion segment. However, in order to acquire a segmental lordotisation it is important to emphasize the need of a correct placement of the Zero- $\mathrm{P}^{\circledR}$ cage - its anterior titanium plate should always follow the anterior vertebral line ${ }^{13}$. During the first 6 postoperative months the global lordotisation was more prominent in female patients after both 1- and 2- level ACDF with anchored cage. Osteoporosis acted as a risk factor for the loss of the surgically-acquired global lordotisation only in term of 12 months after ACDF. Moreover an average value of CoobG/CoobS was significantly higher in patients with this comorbidity even prior to the surgery. Osteoporosis has also no significant impact on GSP development after the implantation of anchored cage ${ }^{15}$. Numerous studies have found no differences in GSP development when comparing the effects of implanted Zero- $\mathrm{P}^{\circledR}$ cage and cage

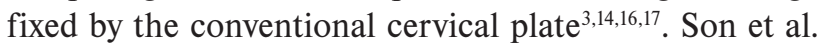
however refer a more significant global lordotisation after the implantation of the anchored cage in comparison to a cage fixed by the conventional plate ${ }^{18}$. CS had no significant impact on the clinical outcome after $\mathrm{ACDF}^{19,20}$. CS occurred most often in ventral portion of motion segment ${ }^{19,20}$. In our study, CS in the ventral portion of motion segment had no significant impact on SSP in term of 24 months after ACDF. However, this result was influenced by the low incidence of CS in our patient cohort (8.2\%).

Segmental lordotisation after 1- and 2- level implantation of the anchored interbody cage was accompanied by an increase in global lordosis in the early postoperative period. A gradual loss of segmental lordotisation occurred during the further postoperative period. The GSP did not follow this trend and an overall increase in global lordosis was recorded. The change in the SSP in one or two cervical motion segments only weakly correlated with the GSP change. The 1- or 2- level ACDF has therefore a limited impact on the GSP development after the surgery ${ }^{3,11,19,21}$.

\section{CONCLUSION}

The change of SSP after the 1- or 2- level ACDF weakly correlates with the GSP change. A significantly greater increase in CobbS was observed during the early postoperative period after 1-level ACDF compared to that after the 2-level ACDF. Osteoporosis, gender, CS and age have no significant impact on the SSP development after 
the surgery. Risk factors associated with the loss of the surgically-acquired global lordotisation are male gender and osteoporosis.

Acknowledgement: This work was supported by project: The application of PACS (Picture Archiving and Communication System) in the research and development, ITMS 26210120004.

Author contributions: RO, MH, PS, RH, BK: performed literature searches and participated in manuscript writing; $\mathrm{RO}, \mathrm{MH}, \mathrm{MB}$ : made revisions and gave final approval of the manuscript; $\mathrm{BK}$ : was responsible for sending the final version.

Conflict of interest statement: Authors state that there are no conflicts of interest regarding the publication of this article.

\section{REFERENCES}

1. Cohen J. Statistical power analysis for the behavioral sciences. 2nd ed. Hillsdale: Lawrance Earlbaum Associates 1998:567.

2. Scholz M, Reyes PM, Schleier P, Sawa A, Baek S, Kandziora F, Marciano $\mathrm{F}$, Crawford N. A new standalone cervical anterior interbody fusion device: Biomechanical comparison with established anterior cervical fixation devices. Spine (Phila Pa 1976) 2009;34:156-60.

3. Vanek P, Bradac O, Saur K. Anterior interbody fusion of the cervical spine with a zero-p spacer: radiographic results with a minimum follow-up of one year in a prospective study. Acta Chir Orthop Traumatol Cech 2011;78(6):562-7.

4. Scholz M, Schnake KJ, Pingel A, Hoffman R, Kandziora F. A new Zeroprofile implant for stand-alone anterior cervical interbody fusion. Clin Orthop Relat Res 2011;469(3):666-73.

5. Azab W, Abdel-Razek M, Ali A, Abdelrahman A, Salaheldin W, Khurram N, Attia H, Soliman D. Outcome evaluation of a Zero-profile implant for anterior cervical discectomy with fusion. Turk Neurosurg 2012;22(5):611-7.

6. Hofstetter CP, Kesavabhotla K, Boockvar JA. Zero-profile anchored spacer reduces rate of dysphagia compared with ACDF with anterior plating. J Spinal Disord Tech 2015;28(5):284-90.

7. Yang $\mathrm{H}$, Chen D, Wang $\mathrm{X}$, Yang L, He H, Yuan W. Zero-profile integrated plate and spacer device reduces rate of adjacent-level ossification development and dysphagia compared to ACDF with plating and cage system. Arch Orthop Trauma Surg 2015;135(6):781-7.

8. Yin M, Ma J, Huang Q, Xia Y, Shen Q, Zhao C, Tao J, Chen N, Yu Z, Ye J, Mo W, Xiao J. The new Zero-P implant can effectively reduce the risk of postoperative dysphagia and complications compared with the traditional anterior cage and plate: a systematic review and metaanalysis. BMC Musculoskeletal Disord 2016;17(1):430.
9. Chen Y, Liu Y, Chen H, Cao P, Yuan W. Comparison of Curvature Between the Zero-P Spacer and Traditional Cage and Plate After 3-Level Anterior Cervical Discectomy and Fusion: Mid-term Results. Clin Spine Surg 2017;30(8):1111-6.

10. Yang $Z$, Zhao Y, Luo J. Incidence of dysphagia of zero-profile spacer versus cage-plate after anterior cervical discectomy and fusion: $A$ meta-analysis. Medicine (Baltimore) 2019;98(25):157-67.

11. Shin JS, Oh SH, Cho PG. Surgical Outcome of a Zero-profile Device Comparing with Stand-alone Cage and Anterior Cervical Plate with Iliac Bone Graft in the Anterior Cervical Discectomy and Fusion. Korean J Spine 2014;11(3):169-77.

12. Cho HJ, Hur JW, Lee JB, Han JS, Cho TH, Park JY. Cervical Stand-alone polyetheretherketone cage versus zero-profile anchored spacer in single-level anterior cervical discectomy and fusion: minimum 2-year assessment of radiographic and clinical outcome. J Korean Neurosurg Soc 2015;58:119-24.

13. Yun DJ, Lee SJ, Park SJ, Oh HS, Lee YJ, Oh HM, Lee SH. Use of a ZeroProfile Device for Contiguous 2-Level Anterior Cervical Diskectomy and Fusion: Comparison with Cage with Plate Construct. World Neurosurg 2017;97:189-98.

14. Vanek P, Bradac O, Delacy P, Lacman J, Benes V. Anterior interbody fusion of the cervical spine with Zero-P spacer: prospective comparative study-clinical and radiological results at a minimum 2 years after surgery. Spine (Phila Pa 1976) 2013;38(13):792-7.

15. Zhang L, Wang J, Feng $X$, Tao Y, Yang J, Wang Y, Zhang S, Cai J. Outcome Evaluation of Zero-Profile Device Used for Single-Level Anterior Cervical Discectomy and Fusion with Osteoporosis Compared without Osteoporosis: A Minimum Three-Year FollowUp Study. World Neurosurg 2018;12(18):1878-8750.

16. Tong MJ, Xiang GH, He ZL, Chen DH, Tang Q, Xu HZ, Tian NF. ZeroProfile Spacer Versus Cage-Plate Construct in Anterior Cervical Diskectomy and Fusion for Multilevel Cervical Spondylotic Myelopathy: Systematic Review and Meta-Analysis. World Neurosurg 2017; 104:545-53.

17. Zhang L, Wang J, Tao Y, Feng X, Yang J, Zhang S. Outcome Evaluation of Zero-Profile Implant Compared with an Anterior Plate and Cage Used in Anterior Cervical Discectomy and Fusion: A Two-Year FollowUp Study. Turk Neurosurg 2016;26(3):416-22.

18. Sun Z, Liu Z, Hu W, Yang Y, Xiao X, Wang X. ZeroProfile Versus Cage and Plate in Anterior Cervical Discectomy and Fusion with a Minimum 2 Years of Follow-Up: A Meta-Analysis. World Neurosurg 2018;120:551-61.

19. Barsa P, Suchomel P. Factors affecting sagittal malalignment due to cage subsidence in standalone cage assisted anterior cervical fusion. Eur Spine J 2007;16:1395-400.

20. Opsenak R, Hanko M, Snopko P, Varga K, Kolarovszki B. Subsidence of anchored cage after anterior cervical discectomy. Bratisl Lek Listy 2019;120(5):356-61.

21. Li Y, Hao D, He B, Wang X, Yan L. The Efficiency of Zero-profile Implant in Anterior Cervical Discectomy Fusion: A Prospective Controlled Long-term Follow-up Study. J Spinal Disord Tech 2015;28(10):398403. 